\title{
Default Mode Network Connectivity Predicts Sustained Attention Deficits after Traumatic Brain Injury
}

\author{
Valerie Bonnelle, ${ }^{1,2}$ Robert Leech, ${ }^{1}$ Kirsi M. Kinnunen, ${ }^{3}$ Tim E. Ham, ${ }^{1}$ Cristian F. Beckmann, ${ }^{1,4}$ Xavier De Boissezon, ${ }^{5,6,7}$ \\ Richard J. Greenwood, ${ }^{8}$ and David J. Sharp ${ }^{1}$ \\ ${ }^{1}$ The Computational, Cognitive and Clinical Neuroimaging Laboratory, Centre for Neuroscience, Division of Experimental Medicine and ${ }^{2}$ The Medical \\ Research Council Clinical Sciences Centre, Imperial College London, London W12 0NN, United Kingdom, ${ }^{3}$ Goldsmiths, University of London, London SE14 \\ 6NW, United Kingdom, ${ }^{4}$ MIRA Institute for Biomedical Technology and Technical Medicine University of Twente, NL-6500 HB Nijmegen, The \\ Netherlands, ${ }^{5}$ INSERM-Imagerie cérébrale et handicaps neurologiques UMR 825, F-31059 Toulouse, France, ${ }^{6}$ Universite de Toulouse, UPS, Imagerie \\ cérébrale et handicaps neurologiques UMR 825, CHU Purpan, Place du Dr Baylac, F-31059 Toulouse Cedex 9, France, ${ }^{7}$ Centre Hospitalier Universitaire de \\ Toulouse, Pole Neurosciences, CHU Purpan, F-31059 Toulouse Cedex 9, France, and ${ }^{8}$ The Institute of Neurology, University College London, London \\ WC1N 3BG, United Kingdom
}

Traumatic brain injury (TBI) frequently produces impairments of attention in humans. These can result in a failure to maintain consistent goal-directed behavior. A predominantly right-lateralized frontoparietal network is often engaged during attentionally demanding tasks. However, lapses of attention have also been associated with increases in activation within the default mode network (DMN). Here, we study TBI patients with sustained attention impairment, defined on the basis of the consistency of their behavioral performance over time. We show that sustained attention impairments in patients are associated with an increase in DMN activation, particularly within the precuneus and posterior cingulate cortex. Furthermore, the interaction of the precuneus with the rest of the DMN at the start of the task, i.e., its functional connectivity, predicts which patients go on to show impairments of attention. Importantly, this predictive information is present before any behavioral evidence of sustained attention impairment, and the relationship is also found in a subgroup of patients without focal brain damage. TBI often results in diffuse axonal injury, which produces cognitive impairment by disconnecting nodes in distributed brain networks. Using diffusion tensor imaging, we demonstrate that structural disconnection within the DMN also correlates with the level of sustained attention. These results show that abnormalities in DMN function are a sensitive marker of impairments of attention and suggest that changes in connectivity within the DMN are central to the development of attentional impairment after TBI.

\section{Introduction}

Consistent goal-directed behavior is dependent on the ability to sustain attention over time. A predominantly right-sided frontoparietal system is often engaged during attentionally demanding tasks (Paus et al., 1997; Coull et al., 1998), and damage to these regions can lead to impairments of sustained attention (Rueckert and Grafman, 1996; Malhotra et al., 2009). However, brain activity within the default mode network (DMN) also tracks fluctuations in attention (Weissman et al., 2006; Hayden et al., 2009), showing reduced activation during most attentionally demanding tasks (Raichle et al., 2001; Buckner et al., 2008).

\footnotetext{
Received March 7, 2011; revised April 14, 2011; accepted April 20, 2011.

Author contributions:V.B., R.L., X.D.B., and D.J.S. designed research;V.B., K.M.K., and T.E.H. performed research; R.L., C.F.B., and R.J.G. contributed unpublished reagents/analytic tools; V.B. and K.M.K. analyzed data; V.B. and D.J.S. wrote the paper.

This work was supported by the Medical Research Council (UK) and the Hammersmith Hospitals Trustees' Research Committee.

This article is freely available online through the J Neurosci Open Choice option.

Correspondence should be addressed to David J. Sharp, Computational, Cognitive and Clinical Neuroimaging

Laboratory, 3rd Floor, Burlington Danes Building, Hammersmith Hospital, Du Cane Road, London W12 0NN, UK.

E-mail: david.sharp@imperial.ac.uk.

DOI:10.1523/JNEUROSCI.1163-11.2011

Copyright $\odot 2011$ the authors $\quad 0270-6474 / 11 / 3113442-10 \$ 15.00 / 0$
}

The DMN consists of a set of brain regions that show highly correlated brain activity and high metabolic demands at rest (Raichle et al., 2001). The posterior cingulate cortex (PCC), precuneus, and parts of the ventromedial prefrontal cortex (vmPFC) are its midline components. These regions have dense white matter connections, suggesting they form part of the brain's structural core (Hagmann et al., 2008). Disruption of the DMN is clinically important, and abnormalities are observed in various neurological and psychiatric disorders (Greicius, 2008). One function of the network is to support internally directed mental activity (Gusnard et al., 2001; Mason et al., 2007). In addition, the DMN is often thought of as a counterpoint to brain regions involved in cognitive control (Seeley et al., 2007). Their interaction may be important for regulating the focus of attention (Hampson et al., 2006; Gilbert et al., 2007; Leech et al., 2011), perhaps promoting a coordinated balance between internally and externally directed thought (Raichle, 2010).

Traumatic brain injury (TBI) patients are particularly impaired at maintaining consistent behavioral performance and provide a unique opportunity to study the neural basis of sustained attention (Stuss et al., 1989; Whyte et al., 1995). These attentional deficits result in part from diffuse axonal injury (DAI) (Scheid et al., 2003), which often affects the structural core of the brain, potentially disrupting the DMN (Povlishock and Katz, 
2005; Hagmann et al., 2008). Previous studies of attentional impairment after TBI have often shown increased activation within the frontoparietal attentional network (Levine et al., 2006; Scheibel et al., 2007; Smits et al., 2009), which have generally been interpreted as a compensatory response to brain injury (Rasmussen et al., 2008; Smits et al., 2009).

Here we use behavioral inconsistency on a simple choice reaction time task (CRT) as a marker of the efficiency of sustained attention after TBI. The neural basis of this vigilance decrement is investigated by analyzing brain activity at the start and end of the task. We use traditional fMRI analysis to explore the functional significance of abnormal brain activation in TBI patients. We then perform complementary analyses of functional and structural connectivity. We investigate whether functional connectivity within the DMN predicts impairments in sustained attention. Finally, we use diffusion tensor imaging (DTI) to investigate whether the impact of DAI on key white matter tracts relate to behavioral impairment. The work demonstrates that abnormal connectivity within the DMN predicts impairments of sustained attention.

\section{Materials and Methods}

Patients: demographic and clinical details. Thirty patients with a history of TBI were recruited, and 28 are included in the reported analyses (22 males; mean age, $36.7 \pm 10.8$; range, $18-54$ years). They were all in the post-acute/chronic phase after injury (mean, 28 months; range, 3-73 months). Injury was secondary to assaults $(36 \%)$, road traffic accidents $(21 \%)$, falls (36\%) and sports-related injury (7\%). Patients were referred to their local TBI service because of persistent cognitive problems. Based on the Mayo classification system for TBI severity (Malec et al., 2007), there were 21 moderate/severe and 7 mild (probable) cases of TBI. This system integrates the duration of loss of consciousness, length of posttraumatic amnesia, lowest recorded Glasgow Coma Scale score in the first $24 \mathrm{~h}$, and initial neuroimaging results. Exclusion criteria were as follows: neurosurgery, except for invasive intracranial pressure monitoring (one patient); history of psychiatric or neurological illness before their head injury; history of significant previous TBI; antiepileptic medication; current or previous drug or alcohol abuse; or contraindication to MRI. The study was approved by the Hammersmith and Queen Charlotte's and Chelsea research ethics committee, and all participants gave written informed consent.

Clinical imaging. All patients except one [who had no initial computed tomography (CT) imaging] had abnormalities either on initial CT imaging or follow-up MRI. Each patient was assessed using standard T1 MRI to assess evidence of focal brain injury and gradient-echo imaging to identify microbleeds, a marker of DAI (Scheid et al., 2003). A senior consultant neuroradiologist reviewed all study MRI scans. At the time of the study, they showed the following: 8 had residual evidence of contusions, 11 had microbleeds as demonstrated on gradient-echo imaging, 3 had evidence of both. Contusions were mainly situated in the inferior parts of the frontal lobes, including the orbitofrontal cortex, and the temporal poles, in a typical lesion distribution for TBI patients (Gentry et al., 1988).

Neuropsychological assessment. A detailed neuropsychological battery was used to assess cognitive function. Current verbal and nonverbal reasoning ability was assessed using Wechsler Abbreviated Scale of Intelligence Similarities and Matrix Reasoning subtests (Wechsler, 1999). Verbal fluency, letter fluency, and color-word (Stroop) tests were administered from the Delis-Kaplan Executive Function System to assess cognitive flexibility, inhibition, and set shifting (Delis et al., 2001). The Trail Making Test (forms A and B) was used to further assess executive functions (Reitan, 1958). Working memory was assessed via the Digit Span subtest of the Wechsler Memory Scale, third edition (WMS-III) (Wechsler, 1997). The Logical Memory I and II subtests of the WMS-III were included as measures of immediate and delayed verbal recall. The people test from the Doors and People test battery was used as a measure of associative learning and recall (immediate and delayed) (Baddeley et al., 1994).
Control groups. Two control groups were used. For the functional imaging study and the DTI analysis, groups of 26 (17 males; mean age, $34.8 \pm 9.6)$ and 28 (20 males; mean age, $37.4 \pm 12.1)$ age-matched healthy volunteers were recruited, respectively. Subjects had no history of neurological or psychiatric disorders.

Choice reaction time task. A simple CRT was used to study sustained attention in the scanner. The task was simple enough to be performed accurately by all the subjects, but demanding enough for patients to show performance decrement [reaction time (RT) increase] as the task progressed. Similar speeded reaction-time tasks have been used in the past to investigate some aspects of sustained attention in both humans (Langner et al., 2010) and animals (Grottick and Higgins, 2002). An initial fixation cross was presented for $350 \mathrm{~ms}$. This was followed by a response cue arrow (go signals, or $)$ ) lasting $1400 \mathrm{~ms}$ and to which subjects were instructed to respond as quickly and as accurately as possible with a right or left index finger press. One hundred and forty-four trials were presented with an interstimulus interval of $1.75 \mathrm{~s}$, which was not jittered, but offset to the TR (2s). Go trials were randomly interleaved with Rest trials, during which the fixation cross remained on the screen (ratio of $5 / 5 / 4$, right/left/rest).

MRI acquisition. MRI data were obtained using a Philips Intera 3.0 Tesla MRI scanner using Nova Dual gradients, a phased array head coil, and sensitivity encoding (SENSE) with an undersampling factor of 2 . High-resolution images (T1-weighted MPRAGE) were acquired with the following acquisition parameters: matrix size $208 \times 208$; slice thickness, $1.2 \mathrm{~mm} ; 0.94 \mathrm{~mm} \times 0.94 \mathrm{~mm}$ in-plane resolution; 150 slices; TR, $9.6 \mathrm{~ms}$; TE, $4.5 \mathrm{~ms}$; flip angle, $8^{\circ}$. Functional MRI images were obtained using a $\mathrm{T} 2{ }^{*}$-weighted gradient-echo echoplanar imaging (EPI) sequence with whole-brain coverage (TR, 2000; TE, 30; 31 ascending slices, $3.25 \mathrm{~mm}$ thick; gap, $0.75 \mathrm{~mm}$; voxel size, $2.5 \times 2.5 \times 5 \mathrm{~mm}$; flip angle, $90^{\circ}$; field of view, $280 \times 220 \times 123 \mathrm{~mm}$; matrix, $112 \times 87$ ). Quadratic shim gradients were used to correct for magnetic field inhomogeneities within the brain. T1-weighted whole-brain structural images were also obtained in all subjects. Paradigms were programmed using Matlab Psychophysics toolbox (Psychtoolbox-3; www.psychtoolbox.org), and stimuli were presented through an IFIS-SA system (In Vivo). Responses were recorded through a fiber-optic response box (Nordicneurolab) interfaced with the stimulus presentation PC running Matlab. Participants had two sessions of imaging: one session consisted of structural brain imaging including DTI, and the other of task fMRI. The average time between the sessions was 1 week.

Standard fMRI analysis of the CRT. A high-level description of how fMRI data was analyzed is presented in Figure 1. Imaging analysis was performed using fMRI Expert Analysis Tool (FEAT, version 5.98), a part of FSL (FMRIB Software Library; www.fmrib.ox.ac.uk/fsl) (Smith et al., 2004). Image preprocessing involved realignment of EPI images, spatial smoothing using a $8 \mathrm{~mm}$ full-width at half-maximum Gaussian kernel, prewhitening using FMRIB's Improved Linear Model (FILM), and temporal high-pass filtering using a cutoff frequency of $1 / 50 \mathrm{~Hz}$. FMRIB's Linear Image Registration Tool (FLIRT) was used to register EPI functional datasets into standard MNI space using the participant's highresolution T1. Two patients were excluded from additional analyses as a result of problems with registration. fMRI data were analyzed using voxelwise time series analysis within the framework of the general linear model (Beckmann et al., 2003). To this end, a design matrix was generated with a synthetic hemodynamic response function and its first temporal derivative. To investigate the impact of sustained attention impairments, brain activation was compared between the first (T1) and the last (T3) third of the task. To this end, four types of events were analyzed: Rest and Go trials during T1 (Rest1 and Go1), and Rest and Go trials during T3 (Rest3 and Go3). The timing of the button press minus $0.2 \mathrm{~s}$ was used as the regressor for Go trials. Rest events were modeled using the trial onset plus $0.35 \mathrm{~s}$. The following contrast images were then generated: Go3-Go1 and Rest3-Rest1. Group effects analysis was performed using FLAME (FMRIB's local analysis of mixed effects). Final statistical images were thresholded using Gaussian random field-based cluster inference with a height threshold of $Z>2.3$ and a cluster significance threshold of $p<0.05$. To control for the possible effect of interindividual differences in gray matter density on the BOLD signal (Oakes et al., 2007), individual gray matter density maps were included in the 


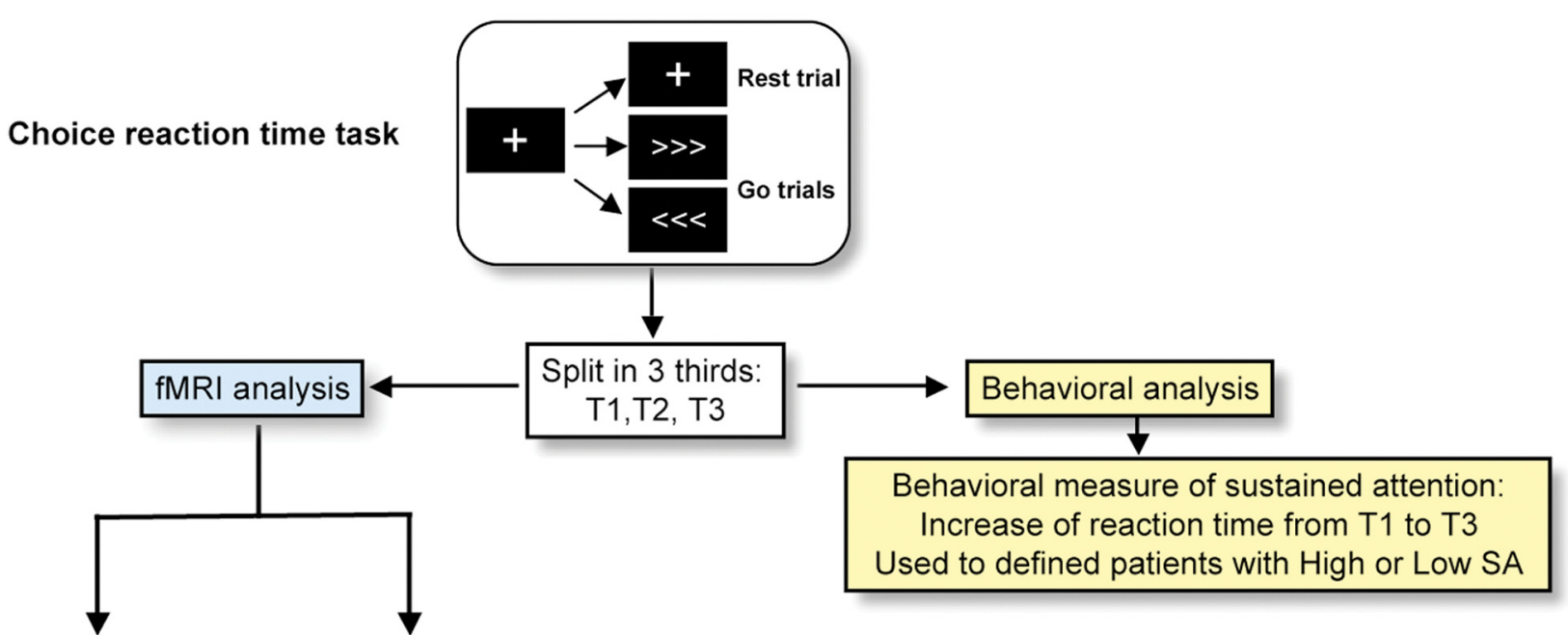

(a)

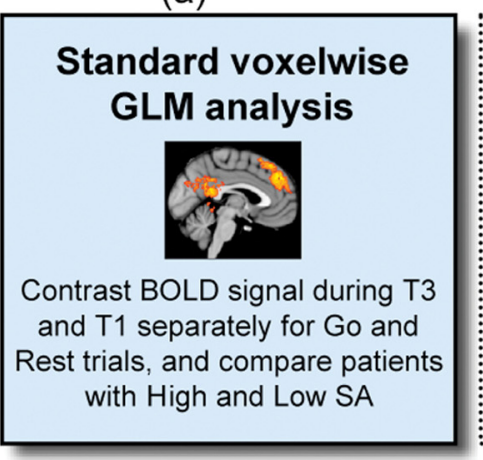

(b)

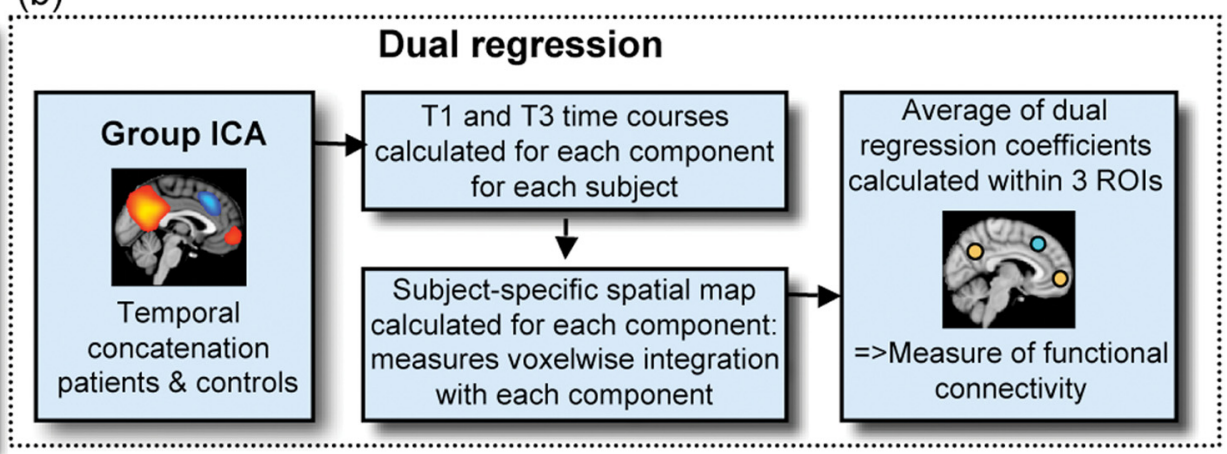

Figure 1. Overview of the methods used to analyze behavioral and brain changes during the performance of the CRT.

FEAT GLM as a confound regressor. Gray matter maps were extracted using FMRIB's automated segmentation tool (FAST), registered in standard space, smoothed to match the fMRI data (Filippini et al., 2009). To further explore the change in activation in the precuneus, a region of interest (ROI) analysis was also performed. Mean percentage BOLD signal change was extracted from a $10 \mathrm{~mm}$ radius sphere centered on the local maxima from the contrast Rest3-Rest1 in patients versus controls (MNI coordinates, $x=-4, y=-68, z=34$ ) (see Table 3).

To investigate further the relationship between brain activation and sustained attention, the patients were split into two subgroups according to their ability to maintain a consistent performance over time. The slowing of RT and increase in error rates as subjects perform a task over time have both been used as markers of reductions in sustained attention (Whyte et al., 1995; Paus et al., 1997; Pattyn et al., 2008; Langner et al., 2010). In patients with brain injury, this type of vigilance decrement may be a particularly useful measure (Malhotra et al., 2009). In our study, although patients maintained the accuracy of their performance, RT decrement was highly variable across individuals. As a result, high- and low-sustained attention (SA) groups were defined on the basis of how much the patient's RT slowed down over the course of the task using a median split of RT changes.

Functional connectivity analysis. For the functional connectivity analyses, we used an independent component analysis (ICA)-based approach (using multivariate exploratory linear decomposition into independent components) (Beckmann and Smith, 2004) in combination with a "dual regression technique" (Filippini et al., 2009; Zuo et al., 2010; Leech et al., 2011). This involved an initial ICA decomposition of the data, followed by further functional connectivity analysis of the output of the ICA. This approach has a number of advantages over the use of seed voxel-based approaches and has been used to probe behavioral and pathology-related differences in multiple populations (Damoiseaux et al., 2008; Filippini et al., 2009).
Temporal concatenation ICA was first used to define 25 reference network maps common to task performance in patients and controls (Beckmann et al., 2005). We then selected a single reference component that captured the previously described DMN, and a set of anticorrelated brain regions described previously as being part of both an executive control network (including the dorsolateral prefrontal cortex) and a salience network (including the anterior cingulate and anterior insular cortices) (Seeley et al., 2007). We refer to this network as the executive control/salience network (EC/SN) (see Fig. 4A). To dissociate the respective effect of DMN and EC/SN functional connectivity, this component was split into its positive (DMN) and negative (EC/SN) parts. These two subcomponents were further analyzed separately. Preprocessed functional data from each individual were split into three equal parts, allowing the analysis of the initial and last thirds of the CRT separately. Subsequent functional connectivity analysis was performed using the dual regression approach (Filippini et al., 2009). This approach allows the derivation of the individual independent component (IC) time courses and spatial maps corresponding to the DMN and EC/SN.

Dual regression proceeds in three steps. In step 1, all unthresholded group maps from the ICA output are linearly regressed against the first and the last third of the preprocessed functional data from each individual (spatial regression). This produces subject-specific time courses of signal fluctuation corresponding to each group-level IC. In step 2, the time courses are variance normalized and then linearly regressed against the corresponding fMRI data (temporal regression), converting each time series into subject-specific spatial maps of the corresponding component (in this case the EC/SN and DMN). This method reliably produces subject-specific approximations to the unthresholded spatial ICs in the group ICA output (Zuo et al., 2010). In step 3, these individuallevel dual-regression components were subsequently used to evaluate individual functional connectivity. We extracted functional connectivity from ROIs within the DMN and EC/SN separately for T1 and T3. Three 
Table 1. Neuropsychological results

\begin{tabular}{|c|c|c|c|c|}
\hline Cognitive variable & Controls (mean \pm SD) & Patients (mean \pm SD) & High SA (mean \pm SD) & Low $S A$ (mean $\pm S D$ ) \\
\hline Similarities raw score & $35.1 \pm 5.7$ & $38.8 \pm 3.9^{* *}$ & $39.5 \pm 2.4^{* *}$ & $38.1 \pm 4.9$ \\
\hline Matrix reasoning raw score & $27.1 \pm 4.5$ & $28.5 \pm 4.6$ & $29.1 \pm 4.2$ & $27.9 \pm 5.15$ \\
\hline Verbal fluency, letter fluency & $47.2 \pm 13.0$ & $42.7 \pm 9.7$ & $44.4 \pm 8.2$ & $41.2 \pm 11.1$ \\
\hline Stroop word reading (s) & $23.0 \pm 4.7$ & $23.0 \pm 5.2$ & $22.0 \pm 4.8$ & $24.07 \pm 5.5$ \\
\hline Stroop inhibition (s) & $24.5 \pm 11.2$ & $57.6 \pm 24.1^{* * *}$ & $47.8 \pm 14.9^{* * *}$ & $67.5 \pm 27.8^{* * * * *}$ \\
\hline Stroop inhibition switching (s) & $50.4 \pm 17.3$ & $69.3 \pm 25.5^{* * *}$ & $64.6 \pm 19.6^{* *}$ & $74.4 \pm 30.6^{* * *}$ \\
\hline Digit Span forward & $11.6 \pm 2.2$ & $10.6 \pm 1.8$ & $10.4 \pm 1.9$ & $10.7 \pm 1.8$ \\
\hline Digit Span backward & $7.6 \pm 1.9$ & $7.3 \pm 2.2$ & $7.8 \pm 2.2$ & $6.8 \pm 2.2$ \\
\hline Logical Memory I 1st recall total & $27.1 \pm 8.5$ & $27.9 \pm 6.7$ & $27.3 \pm 7.9$ & $28.5 \pm 5.4$ \\
\hline Logical Memory I recall total & $43.8 \pm 12.6$ & $45.9 \pm 8.4$ & $45.1 \pm 9.6$ & $46.6 \pm 7.2$ \\
\hline Logical Memory II recall total & $26.3 \pm 8.1$ & $29.2 \pm 7.8$ & $29.4 \pm 7.0$ & $29.0 \pm 8.8$ \\
\hline People test immediate total & $28.6 \pm 6.0$ & $24.1 \pm 4.9^{* *}$ & $22.8 \pm 4.2^{* * *}$ & $25.5 \pm 5.4$ \\
\hline
\end{tabular}

Neuropsychological results for high- and low-SA TBI patient groups compared to an age-matched control group. Significant differences between TBI patient groups are shown by ${ }^{p} p<0.05$. Significant differences between patients and controls are shown by ${ }^{* *} p<0.05$ and ${ }^{* * *} p<0.01$. The color-naming, word-reading, inhibition/switching, and letter fluency tests are from the Delis-Kaplan Executive Function System.

ROIs were defined using the peaks of functional connectivity local maxima from the DMN and EC/SN components. They corresponded to the two major DMN nodes (MNI coordinates, precuneus, $6,-62,28$; vmPFC, $-2,54,-8)$ and a region of the EC/SN where patients with sustained attention impairment showed increased activation over time [anterior cingulate cortex (ACC), -2, 22, 40]. The measures derived from this analysis index the strength of functional connectivity from each region to the rest of its network during T1 and T3 separately. For instance, the measure plotted on Figure $4 B$ represents the functional connectivity of the precuneus to the rest of the DMN during T1, controlling for age. The average gray matter densities of the DMN and EC/SN were extracted from individual gray matter density maps used previously as confound regressors in the FEAT analysis. To control for individual variability in gray matter density, these measures were regressed-out of the functional connectivity analyses.

Structural connectivity: DTI. Diffusion-weighted volumes with gradients applied in 64 noncollinear directions were collected. The following parameters were used: 73 contiguous slices; slice thickness, $2 \mathrm{~mm}$; field of view, $224 \mathrm{~mm}$; matrix, $128 \times 128$ (voxel size, $1.75 \times 1.75 \times 2 \mathrm{~mm}^{3}$ ); $b=$ 1000; and four images with no diffusion weighting $\left(b=0 \mathrm{~s} / \mathrm{mm}^{2}\right)$. Diffusion-weighted images were registered to the $b=0$ image by affine transformations to minimize distortion caused by motion and eddy currents, and then brain extracted using BET (Brain Extraction Tool) (Smith, 2002), part of the FSL image-processing toolbox (Smith et al., 2004). Voxelwise fractional anisotropy (FA) and mean diffusivity (MD) maps were generated using FMRIB's Diffusion Toolbox in FSL. A region of interest approach was taken, focusing on the two cingulum bundles that connect the precuneus/PCC and vmPFC nodes in the DMN. In addition, we studied the right superior longitudinal fasciculus (SLF) to investigate whether white matter damage in the right frontoparietal attentional system was correlated with sustained attention impairment. Probabilistic maps of these tracts were derived from the Johns Hopkins University ICBM-DTI-81 white-matter labels atlas available in FSL (Mori et al., 2005). We did not use probabilistic tractography to define individual tracts because of the inherent difficulty in interpreting the output of tractography in patient groups with white matter disruption (Hua et al., 2008). The tracts were binarized, translated to each subject's individual diffusion space using FLIRT, thresholded at 0.5 , and rebinarized. The aim was to include only the core of the tract, while excluding peripheral parts of the fiber tract that show pronounced interindividual variability. FA and MD values were then extracted from the tracts of interest.

Voxel-based morphometry analysis. To test for group differences in gray matter density, we also conducted a voxel-based morphometry (VBM) analysis. Structural data were analyzed with FSL-VBM, a VBM style analysis (Ashburner and Friston, 2000; Good et al., 2001) performed with FSL tools (Smith et al., 2004). First, structural images were brain extracted
Table 2. Behavioral results

\begin{tabular}{lccc}
\hline & Whole task & T1 & T3 \\
\hline TBI patients & & & \\
$\quad$ Mean RT (ms) & $461.3 \pm 18.2^{*}$ & $447.7 \pm 18.3$ & $472.2 \pm 19.1^{*, * * *}$ \\
IIV & $0.189 \pm 0.011^{*}$ & $0.169 \pm 0.0117$ & $0.195 \pm 0.014^{* *}$ \\
\% Accuracy & $96.4 \pm 1.1^{*}$ & $96.0 \pm 0.9^{*}$ & $96.5 \pm 0.5^{*}$ \\
Controls & & & \\
Mean RT (ms) & $417.6 \pm 7.4$ & $431.9 \pm 7.6$ & $419.4 \pm 7.2$ \\
IIV & $0.154 \pm 0.007$ & $0.153 \pm 0.010$ & $0.148 \pm 0.008$ \\
\% Accuracy & $98.1 \pm 0.2$ & $98.3 \pm 0.4$ & $98.2 \pm 0.4$ \\
\hline
\end{tabular}

Behavioral results for TBI patients and controls on the whole CRT and for T1 and T3 separately. Significant differences between patients and controls shown by ${ }^{*} p<0.05$ and ${ }^{* *} p<0.01$. Significant differences between the first and last parts shown by ${ }^{* *} p<0.01$.

using BET (Smith, 2002). Next, tissue-type segmentation was performed using FAST4 (Zhang et al., 2001). The resulting gray-matter partial volume images were then aligned to MNI 152 standard space using the affine registration tool FLIRT (Jenkinson and Smith, 2001). Images were then averaged to create a study-specific template, to which the native gray matter images were then nonlinearly reregistered. The registered partial volume images were then modulated (to correct for local expansion or contraction) by dividing by the Jacobian of the warp field. The modulated segmented images were smoothed with an isotropic Gaussian kernel with a $\sigma$ value of $3 \mathrm{~mm}$ and thresholded at a nominal $t$ value of 2.3. Finally, a voxelwise GLM was applied using permutation-based nonparametric testing, correcting for multiple comparisons across space. We used two distinct design matrices to compare patients with all controls, and then patients with low versus high sustained attention.

\section{Results}

\section{Behavior}

Our patients had a cognitive profile typical of TBI (Millis et al., 2001), with impairments of information processing speed, executive function, and associative learning and memory (Table 1). The patients performed the CRT accurately ( $>96 \%$ correct), although with slower RT and more intraindividual variability (IIV) than controls (Table 2), both of which are hallmarks of attentional problems after TBI (Stuss et al., 1989; Whyte et al., 1995). As a group, the patients were unable to maintain consistent performance over time, as demonstrated by a comparison of RT between the first (T1) and last thirds (T3) of the task. A time by group mixed ANOVA showed a significant interaction $\left(F_{(1,52)}=\right.$ 8.076; $p=0.006)$, resulting from patients' responses at T3 being significantly slower than at $\mathrm{T} 1(t=-4,51 ; \mathrm{df}=28 ; p<0.001)$ 
and also slower than those of controls at $\mathrm{T} 3(t=-2.52 ; \mathrm{df}=53 ; p=0.015)$ (Table $2)$. Separate ANOVAs were also performed for IIV and accuracy. These showed a significant effect of group in both cases, but no interaction. Patients showed more $\operatorname{IIV}\left(F_{(1,52)}=5.126 ; p=0.028\right)$ and marginally lower accuracy than controls $\left(F_{(1,52)}=\right.$ $6.625 ; p=0.013)$.

Our measure of sustained attention ability, RT change, correlated with other measures of attention and executive function. First, there was a significant correlation with the overall IIV ( $p=0.015 ; r=0.455)$, a measure known to reflect aspects of sustained attention deficits in TBI patients (Stuss et al., 1989). Second, when considering IIV during T1 and T3 separately, RT slowing showed no correlation with IIV at T1, but was correlated with IIV at T3 (one-tailed $p=0.037 ; r=0.342$ ), suggesting a relationship between vigilance decrement, as measured by change in RT, and the moment-to-moment lapses of attention that combine to produce a high IIV. Third, the change in RT also correlated with a neuropsychological measure of inhibition (Stroop inhibition RT), which has been suggested to be tightly coupled with sustained attention (one-tailed $p=$ 0.03; $r=0.358$ ) (Robertson et al., 1997;

Fernandez-Duque and Posner, 2001). These relationships suggest that the measure of RT change is useful as a general marker of sustained attention deficits in our patients.

\section{Neuroimaging}

Whole-brain event-related analysis of the CRT revealed a predictable pattern of activation within bilateral sensory, motor, and superior parietal regions, as well as within the supplementary motor area, occipital regions, and the thalami, and left insula. As expected, deactivation was observed within the DMN, with a peak deactivation in the vmPFC. Over the whole run, task-related activation and deactivation was similar for patients and controls, suggesting an absence of general, nonspecific changes in brain physiology after TBI.

\section{Default mode network activation increases over time in patients}

We expected that impairments in sustained attention would be accompanied by abnormal activation within networks involved in supporting attentional processing. Initially, we investigated this by comparing brain activation at T1 with that at T3, where patients showed abnormally variable performance (see Fig. 1 for overview of methods). Rest and Go trials were analyzed separately. In general there were increases of activation at T3 for both groups. In controls, T3 Go trials were associated with significantly greater activation within the right inferior frontal gyrus (IFG) and insula. Patients showed a distinct and more extended increase on Go trials within parts of the DMN, including the precuneus, vmPFC, and bilateral inferior parietal lobes. There were no regions that were less activated at T3 than T1. For Rest trials, only patients showed increased activation from T1 to T3. This was observed particularly within the DMN, with peaks of
Table 3. Whole brain analysis local maxima

\begin{tabular}{|c|c|c|c|c|}
\hline & \multicolumn{4}{|c|}{ MNI coordinates } \\
\hline & Zscore & $x$ & $y$ & $z$ \\
\hline \multicolumn{5}{|l|}{ Patients vs controls } \\
\hline \multicolumn{5}{|l|}{ G03 vs G01 } \\
\hline L frontal pole & 4.32 & 18 & 46 & 38 \\
\hline$R$ medial frontal gyrus & 3.88 & 4 & 54 & 16 \\
\hline L superior frontal gyrus & 3.86 & -2 & 34 & 54 \\
\hline \multicolumn{5}{|l|}{ Rest3 vs Rest1 } \\
\hline Brain stem & 4.5 & 0 & -26 & -24 \\
\hline L precuneus & 4.46 & -4 & -68 & 34 \\
\hline L temporal pole/parahippocampal gyrus & 4.35 & -32 & 4 & -26 \\
\hline R medial frontal gyrus & 4.42 & 8 & 58 & 14 \\
\hline R superior frontal gyrus & 3.75 & 4 & 32 & 46 \\
\hline L superior frontal gyrus & 3.59 & -14 & 36 & 36 \\
\hline \multicolumn{5}{|l|}{ Low-SA vs high-SA patients } \\
\hline \multicolumn{5}{|l|}{ G03 vs G01 } \\
\hline L inferior frontal gyrus & 5.25 & -52 & 30 & 10 \\
\hline L superior frontal gyrus & 5.21 & -4 & 44 & 34 \\
\hline L posterior cingulate cortex & 4.42 & -2 & -46 & 18 \\
\hline L inferior parietal lobe & 4.7 & -50 & -66 & 34 \\
\hline \multicolumn{5}{|l|}{ Rest 3 vs Rest1 } \\
\hline L precuneus & 4.72 & -8 & -74 & 30 \\
\hline L posterior cingulate cortex & 4.57 & -2 & -44 & 24 \\
\hline
\end{tabular}

Local maxima of brain activations for individual clusters from the comparisons of patients and controls, and the comparison of the two groups of patients (high vs low SA). Coordinates are given for brain activation in $\mathrm{T} 3>\mathrm{T} 1 \mathrm{for}$ $\mathrm{Go}$ (G03 vs G01) and Rest (Rest3 vs Rest1) trials, with associated Z values. Activation within a cluster may extend into other cortical and subcortical areas. R, Right; L, left.

activation in the vmPFC, precuneus, and left inferior parietal lobe. Increased activation was also observed in the left IFG.

We next directly compared patients and controls. For Go trials, patients showed a greater increase in activation than controls from $\mathrm{T} 1$ to $\mathrm{T} 3$ in the medial PFC, with a peak of activation within the rostral superior frontal gyrus (Fig. 2; Table 3). For Rest trials, 


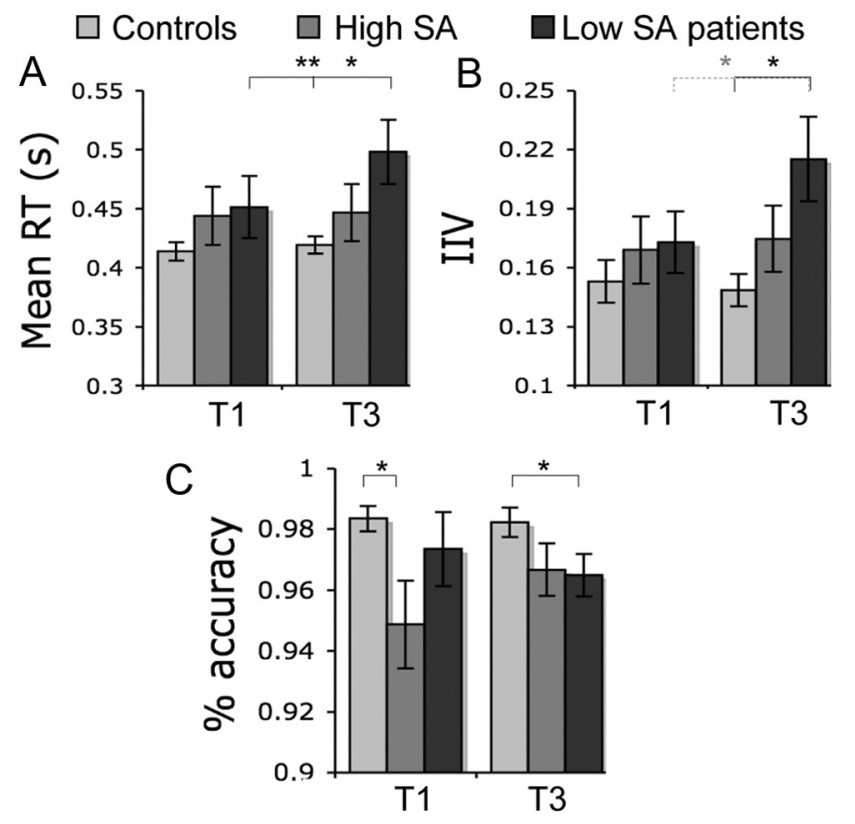

Figure 3. Behavioral results for the first (T1) and the last (T3) third of the choice reaction task. Controls (light gray), high-SA TBI patients (middle gray), and low-SA patients (dark gray) are shown. $\boldsymbol{A}$, Mean reaction time in seconds. $\boldsymbol{B}, \mathrm{IIV} \pm$ SEM. $\boldsymbol{C}$, Percentage accuracy \pm SEM. ${ }^{* *} p<0.001 ;{ }^{*} p<0.05$; Statistical significance. Light gray asterisk refers to $p<0.1$ difference between low-SA patients at T1 and T3.

patients showed extensive increases in activation between $\mathrm{T} 1$ and T3 in parts of the DMN including the precuneus, the PCC, the vmPFC, and the left parahippocampal gyrus (Fig. 2; Table 3). Increased activation was also seen in the superior frontal gyri and the brain stem. This was the result of patients showing increased activation from T1 to T3 (Fig. 2C).

\section{Impairments of sustained attention after TBI are associated with increased DMN activation over time}

Behavior in high-and low-SA groups

For the purposes of our imaging analysis, we defined two sustained attention groups by splitting the patients into those with low and high RT change between T1 and T3. This allowed us to directly address heterogeneity within the patients group, by comparing patients with high and low SA.

An ANOVA comparing the groups' RT at T1 and T3 showed a significant time by group interaction $\left(F_{(1,26)}=43.192 ; p<\right.$ 0.0005 ) (Fig. 3). As was highly likely, the low-SA group showed a significant increase in RT between $\mathrm{T} 1$ and $\mathrm{T} 3(t=-7.75 ; \mathrm{df}=13$; $p<0.0005)$. In contrast, the performance of the high-SA group was stable over time. We also compared the two patient groups with controls. The low-SA group had higher RT than controls at T3 $(t=-3.578 ; \mathrm{df}=38 ; p=0.001)$. In contrast, there was no difference between the high-SA patients and controls.

An ANOVA comparing IIV in the high- and low-SA groups showed no group by time interaction, although in the low-SA group there was a trend toward an increase in IIV between T1 and T3 $(t=-1.79 ; \mathrm{df}=13 ; p=0.096)$ that was not present in the high-SA group $(p>0.45)$. In addition, low-SA patients had higher IIV than controls at T3 $(t=-3.469 ; \mathrm{df}=38 ; p=0.001)$. Again, high-SA patients and controls were not significantly different. Accuracy was not significantly different between the high- and low-SA groups. Comparing the two patient groups to controls, the high-SA group performed less accurately at T1 than controls $(t=2.90 ; \mathrm{df}=38 ; p=0.006)$, whereas the low-SA group performed less accurately than controls at T3 $(t=2.09$; $\mathrm{df}=38 ; p=0.043$ ).

Neuroimaging results for high-and low-sustained attention groups

The two patient groups showed a different pattern of brain activation within the DMN. The low-SA group showed a greater increase in activation within the PCC from T1 to T3 than the high-SA group in both Rest and Go trials (Fig. 2, Table 3). On Rest trials, this difference extended into the precuneus, and on Go trials, it included additional areas of increased activation in the left inferior parietal lobe, another node in the DMN, as well as in the left rostral superior frontal gyrus. This difference was the result of increased activation from T1 to T3 in the low-SA group (Fig. 2D). No regions showed decreased activation at the end of the task. The most consistent change in activation over time was observed within the precuneus/PCC. Over the whole patient group, the magnitude of activation change within the precuneus ROI on Rest trials (see Materials and Methods) correlated positively with the change in RT over time $(r=0.572 ; p=0.002)$. Within this region, the relationship was not observed on Go trials, and was also not seen in controls for either Rest or Go trials.

Importantly, in most other ways the two patient groups were very similar. The groups showed equivalent RT, IIV, and accuracy at $\mathrm{T} 1$, demonstrating that both were initially performing the task at a similar level. The neuropsychological assessment showed that the low-SA group had more impairment of executive function, but in other ways was very similar to the high-SA group (Table 1). In addition, the two groups had comparable injury severities according to the Mayo classification (low SA, 4 mild and 10 moderate to severe; high SA, 3 mild and 11 moderate to severe) and similar times since their injury (low SA, $22 \pm 21$ months; high SA, $14 \pm 10$ months). There was also no significant difference in the presence (low SA, 5; high SA, 6) or number of microbleeds (low SA, $6 \pm 4$; high SA, $13 \pm 12$ ). Only four patients in each group had focal lesions visible on $\mathrm{T} 1$, and the average size of these lesions was not significantly different (low SA, $8250 \pm$ 4115 voxels; high SA, $6065 \pm 6405$ voxels). When the four patients with lesions were removed from the low-SA group, there was still a significant increase in RT between T1 and T3 in this group of patients $(t=8.844 ; \mathrm{df}=8 ; p<0.0005)$. Furthermore, the high- and low-SA groups did not differ in age (low SA, $35 \pm 9$; high SA, $38 \pm 12$ ), gender (low SA, 12 males; high SA, 10 males), and years of education ( $\sim 15$ years in each group). Finally, the VBM analysis comparing gray matter density showed no difference between the two groups.

\section{Functional connectivity}

We extended the standard fMRI approach using a dual regression analysis that provides a voxel-based measure of network functional connectivity for each individual and controls for the effects of neural activity in other brain regions (Fig. 1) (Filippini et al., 2009; Zuo et al., 2010). We selected a single component from a combined ICA in controls and patients for further analysis. This included the DMN as well as a set of anticorrelated regions (Fig. $4 A$ ). The DMN component included the precuneus and vmPFC, as well as the inferior parietal lobes, middle temporal and frontal gyri, thalami, and parahippocampal gyri bilaterally. The anticorrelated EC/SN network included parts of the executive control network described previously (bilateral dorsolateral prefrontal cortices and supramarginal gyri) and salience network (ACC and bilateral insulae) (Seeley et al., 2007). Given the findings from the standard fMRI analysis described above, we examined functional 
connectivity of selected regions of interest within the precuneus and ventromedial PFC in the DMN and the ACC in the EC/SN (see Materials and Methods). The measures derived from this analysis index the functional connectivity of each region of interest to the rest of its network (DMN or $\mathrm{EC} / \mathrm{SN}$ ).

Low functional connectivity within the DMN predicts impairment in sustained attention after TBI

We were particularly interested in investigating whether functional connectivity within the DMN could predict sustained attention ability. Within the patient group, precuneus functional connectivity at T1 was strongly correlated with the change in RT over time (Spearman $r=$ $-0.498 ; p=0.008)$, a relationship that was not seen in controls (Fig. $4 B$ ). This correlation remained significant when the eight patients with focal lesions were removed from the analysis (Spearman's $r=$ $-0.691 ; p=0.001)$, confirming that it was not an artifact of the analysis of patients with focal lesions. There was no significant relationship between $\mathrm{T} 1$ functional connectivity of the vmPFC within the DMN or the ACC within the EC/SN and change in RT over time.

To test whether functional connectivity predicts behavior, we performed a stepwise logistic regression analysis into which the measures of functional connectivity of the precuneus, vmPFC, and ACC with their respective networks at $\mathrm{T} 1$ were entered. Only the precuneus was included in the resulting model $\left(\chi^{2}=5.0 ; \mathrm{df}=1 ; p=0.025\right)$, which was able to correctly classify patients into high- and low-SA groups with $63 \%$ accuracy. The predictive value of functional connectivity was not related to the presence of focal cortical lesions: when we removed the eight patients with focal lesions, the classification accuracy of the model improved to $69 \%\left(\chi^{2}=5.6 ; \mathrm{df}=1 ; p=\right.$ $0.018)$.

\section{Voxel-based morphometry results}

As expected, the direct comparison of patients and controls showed a number of areas with significantly reduced gray matter density in the patients group. Differences were observed in bilateral insular and central opercular regions, the parahippocampal gyri, the temporal poles, the putamens, the right inferior temporal gyrus, and the left inferior frontal gyrus. No significant difference was observed between the two patients subgroups.

The structure of the right cingulum bundle is correlated with sustained attention ability

We next investigated whether damage to the structural connections within the DMN is related to sustained attention impairment. We studied the theoretically selected right and left cingulum bundles, which connect the posterior and anterior parts of the DMN (Greicius et al., 2009) (Fig. 5A). In addition, we investigated the right SLF, which connects parts of the right frontoparietal "attention" network (Schmahmann and Pandya,
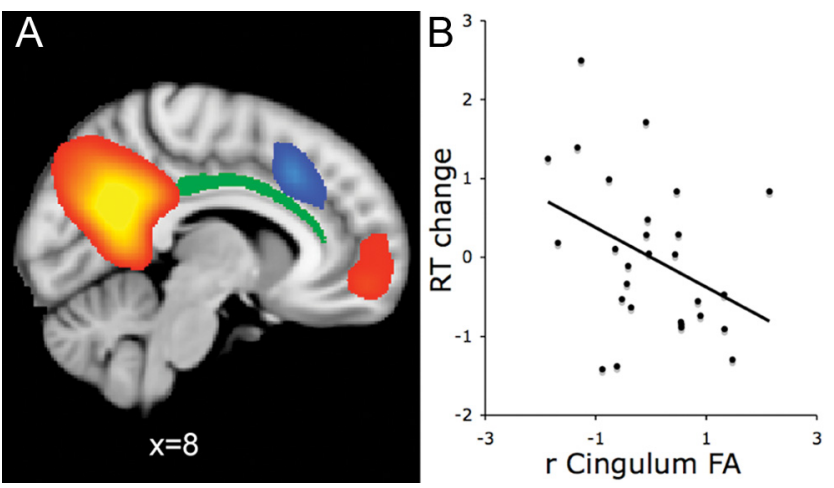

Figure 5. The structure of the right cingulum bundle is correlated with sustained attention impairment. $\boldsymbol{A}$, Sagittal view $(x=4)$ of the reference component selected for the functional connectivity analysis (Fig. 4). Anterior and posterior midline nodes of the DMN are shown in warm colors, and the midline node within the EN is shown in blue. The right cingulum bundle connecting the posterior and anterior parts of the DMN is shown in green. $\boldsymbol{B}, F A$ of the cingulum bundle in patients is plotted against the change in RT between the first and the last part of the task $(N=28)$. Measures are age normalized, i.e., age was regressed out from the measures using a linear regression, where residuals were saved as standardized values.

2006). Our previous work has demonstrated widespread white matter damage in a similar patient group using DTI (Kinnunen et al., 2010). As expected, patients showed lower FA and higher MD than controls. FA was lower in patients compared to controls in the right $(t=2.404 ; \mathrm{df}=54 ; p=0.02)$ and left cingulum bundles $(t=2.419 ; \mathrm{df}=54 ; p=0.019)$, but not in the right SLF. MD was 
significantly higher in patients for all three tracts (right cingulum, $t=-2.216, \mathrm{df}=54, p=0.031$; left cingulum, $t=-4.216, \mathrm{df}=$ $54, p<0.0005$; right SLF, $t=-4.302, \mathrm{df}=54, p<0.0005$ ).

White matter integrity within the right cingulum bundle showed a significant correlation with RT change between $\mathrm{T} 3$ and $\mathrm{T} 1$. Patients with greater sustained attention deficits (high RT change) had lower FA in this tract (Spearman's $r=-0.397 ; p=0.037$ ) (Fig. 5B). No significant relationship was found between sustained attention and FA or MD in the two other tracts investigated.

\section{Discussion}

TBI frequently produces impairments of sustained attention. These can manifest as a failure to maintain consistent goaldirected behavior over time (Stuss et al., 1989; Whyte et al., 1995). Here we show that this type of cognitive impairment is associated with abnormalities within the DMN. The ability to regulate DMN activity appears to be a central part of normal brain function, with greater relative deactivation seen during more cognitively demanding tasks (Singh and Fawcett, 2008; Pyka et al., 2009; Zhang and $\mathrm{Li}, 2010$ ). We show that impairments of sustained attention after TBI, as revealed by behavioral inconsistency, are associated with an increase in DMN activity. Furthermore, despite the absence of any initial performance difference between high- and low-functioning patients, initial functional connectivity within the DMN predicts the development of sustained attention deficits. The presence of focal brain injury or differences in gray matter density does not explain our findings, as DMN functional connectivity predicts performance in a subgroup of patients without focal lesions. Instead, white matter damage in key tracts appears to be critical. Using DTI, we show that greater structural disconnection within the DMN is also associated with impairments of sustained attention. Our results demonstrate that abnormalities in DMN function are a sensitive marker of impairments of attention and support the conclusion that changes in connectivity within the DMN are central to the development of attentional impairment after TBI.

We defined our two patient groups based on a measure of performance decline over time (vigilance decrement). This is distinct from moment-to-moment changes in attention (vigilance level) (Sarter et al., 2001). Both have been used to study sustained attention, and vigilance decrement appears to be a particularly sensitive marker after brain injury (Whyte et al., 1995; Rueckert and Grafman, 1996; Malhotra et al., 2009). The task we used was deliberately simple to ensure that all patients were able to perform it accurately. This had important advantages. First, it ensured that accuracy was balanced between the high- and low-SA groups, an important consideration in functional imaging studies (Price et al., 2006). Second, the design allowed us to focus on a common clinical problem. TBI patients may perform tasks well initially, but often find it difficult to maintain their attentional focus, leading to impaired performance over time (Stuss et al., 1989). By using a simple repetitive task, we were able to reproduce this problem without including additional cognitive complexity inherent in many standard cognitive assessments of attention and executive functions (Robertson et al., 1997).

An important issue in determining patients' outcomes after TBI is that similar severities of injury lead to highly variable cognitive outcomes (Lowenstein, 2009). Impairments of sustained attention are a key problem, and we show that connectivity of the DMN predicts variability in sustained attention impairment. This is unlikely to be a nonspecific effect of injury severity. The high- and low-SA groups were well balanced in terms of clinical injury severity, general cognitive function, and focal lesion vol- ume. Indeed, our predictive model improved when a subgroup without focal lesions was analyzed, suggesting that the result is not secondary to artifacts in the image analysis that might be produced by the presence of focal lesions.

In healthy individuals, the failure to suppress activity in the PCC and precuneus is associated with momentary attentional lapses, as indicated by trial-to-trial increases in reaction times (Weissman et al., 2006). Converging evidence is provided by single cell recordings from monkeys' PCC, which similarly show that increases in the firing rate of PCC neurons just before a monkey's response are associated with RT slowing and increased error rates (Hayden et al., 2009). Over a longer time period, we demonstrate that increased activity in the same part of the DMN predicts which patients show slower reaction times as the task progresses. Together, these results suggest that increasing activity in the precuneus and PCC is associated with a shift away from sustained and focused goal-directed behavior. Previous studies of TBI patients have reported increased activation of the PCC and precuneus during attentionally demanding tasks (Levine et al., 2006; Rasmussen et al., 2008; Smits et al., 2009). This has often been interpreted as reflecting a compensatory neural process (Caeyenberghs et al., 2009; Kim et al., 2009). Our results suggest that this is not the case. Rather, increased activation within the DMN reflects a failure to maintain goal-directed attentional focus.

The importance of the DMN to sustaining attention is also emphasized by our functional connectivity results. These complement the traditional fMRI analysis in two main ways. First, high-level cognitive functions such as attention depend upon the integrated functioning of distributed brain networks (Kim et al., 1999; Kelly et al., 2008). Therefore, focusing only on relative changes of the activation in single brain regions is unlikely to provide a complete description of network function. Second, our traditional approach involved the comparison of DMN activity at different time points. This relative measure cannot provide predictive information about changes in behavior that evolve over the same time period. In contrast, by using dual regression to investigate DMN functional connectivity in distinct phases of task performance, we obtain regionally specific yet integrated network measures, which are independent of the changes in sustained attention over time.

Patients with low initial levels of functional connectivity showed more RT increase over time. Importantly, the low- and high-SA groups had similar initial behavioral performance. Therefore, the predictive information about subsequent performance did not simply reflect behavioral performance at T1. The DMN shows highly reactive changes that reflect cognitive load (Singh and Fawcett, 2008; Zhang and Li, 2010). Hence, as low-SA patients showed lower functional connectivity, the initial difference in functional connectivity is likely to reflect the response of the DMN to a greater cognitive load associated with the task performance at T1. To perform efficiently, cognitively impaired TBI patients are forced to exert more top-down attentional control (Azouvi et al., 2004; Rasmussen et al., 2008). We suggest that this control results initially in suppressed functional connectivity from the precuneus/PCC to the rest of the DMN, a change accompanied by a failure of consistent DMN regulation over time.

Our results are to some extent compatible with both main cognitive theories of vigilance decrement. Higher cognitive load for impaired patients at the start of the task could result in a decreased attentional capacity over time because of mental fatigue. This has been suggested as a cause of vigilance decrement, as attentional resources are temporarily depleted by overuse 
(Lisper and Tornros, 1974; Grier et al., 2003; Langner et al., 2010). An alternative proposal is that vigilance decrement results from a failure to maintain attentional focus on a task because of a withdrawal of top-down control, perhaps associated with underarousal, but not necessarily attributable to resource depletion (Robertson et al., 1997; Manly et al., 1999; Pattyn et al., 2008). As top-down control declines, patients would be expected to show less control of their responses and more distractibility, which has been demonstrated previously (Robertson et al., 1997). Increased activation of the precuneus/PCC and other regions of the DMN have been linked to internally focused attention and mind wandering (Buckner et al., 2008), so our results are also compatible with this hypothesis. The effects of brain injury on mental fatigue and mind wandering are likely to be interrelated (Langner et al., 2010), and one interpretation of our results is that increased initial cognitive load depletes attentional resources, which subsequently makes it more difficult to exert top-down control, leading to mind wandering and less focused behavior.

The pattern of focal cortical damage we observed cannot explain our functional imaging results, as the relationship between functional connectivity and sustained attention was still present in a subgroup of patients with no focal brain injury, and our VBM analysis showed no difference in gray matter volume. Instead our results highlight the importance of structural disconnection secondary to DAI in the development of attentional impairment. The cingulum bundle connects the anterior and posterior midline nodes within the DMN (Greicius et al., 2009), and its structure has been investigated previously using DTI (van den Heuvel et al., 2008; Greicius et al., 2009). In the normal brain, the right cingulum bundle structure correlates with individual differences in attention (Takahashi et al., 2010). We showed that after TBI, reduced FA in the right cingulum bundle is associated with impairments of sustained attention. As expected, our patients showed evidence of widespread axonal damage. However, the relationship with sustained attention was specific to the right cingulum bundle, emphasizing the importance of this tract for this process.

Our results emphasize the importance of the precuneus and PCC as nodes in a system involved in the regulation of attention. Although these regions are rarely focally damaged, they appear to be vulnerable to the effects of structural disconnection, perhaps because of their dense connectivity (Hagmann et al., 2008). We provide evidence that DMN function predicts sustained attention impairments after TBI, and that disconnection within this network is likely to be the underlying structural cause.

\section{References}

Ashburner J, Friston KJ (2000) Voxel-based morphometry-the methods. Neuroimage 11:805-821.

Azouvi P, Couillet J, Leclercq M, Martin Y, Asloun S, Rousseaux M (2004) Divided attention and mental effort after severe traumatic brain injury. Neuropsychologia 42:1260-1268.

Baddeley AD, Emslie H, Nimmo-Smith I (1994) Doors and people test: a test of visual and verbal recall and recognition. Bury-St-Edmunds, UK: Thames Valley Test Company.

Beckmann CF, Smith SM (2004) Probabilistic independent component analysis for functional magnetic resonance imaging. IEEE Trans Med Imaging 23:137-152.

Beckmann CF, Jenkinson M, Smith SM (2003) General multilevel linear modeling for group analysis in FMRI. Neuroimage 20:1052-1063.

Beckmann CF, DeLuca M, Devlin JT, Smith SM (2005) Investigations into resting-state connectivity using independent component analysis. Philos Trans R Soc Lond B Biol Sci 360:1001-1013.

Buckner RL, Andrews-Hanna JR, Schacter DL (2008) The brain's default network: anatomy, function, and relevance to disease. Ann N Y Acad Sci 1124:1-38.
Caeyenberghs K, Wenderoth N, Smits-Engelsman BC, Sunaert S, Swinnen SP (2009) Neural correlates of motor dysfunction in children with traumatic brain injury: exploration of compensatory recruitment patterns. Brain 132:684-694.

Coull JT, Frackowiak RS, Frith CD (1998) Monitoring for target objects: activation of right frontal and parietal cortices with increasing time on task. Neuropsychologia 36:1325-1334.

Damoiseaux JS, Beckmann CF, Arigita EJ, Barkhof F, Scheltens P, Stam CJ, Smith SM, Rombouts SA (2008) Reduced resting-state brain activity in the "default network" in normal aging. Cereb Cortex 18:1856-1864.

Delis DC, Kaplan E, Kramer JH (2001) Delis-Kaplan executive function system. San Antonio, TX: Psychological Corporation.

Fernandez-Duque D, Posner MI (2001) Brain imaging of attentional networks in normal and pathological states. J Clin Exp Neuropsychol 23:74-93.

Filippini N, MacIntosh BJ, Hough MG, Goodwin GM, Frisoni GB, Smith SM, Matthews PM, Beckmann CF, Mackay CE (2009) Distinct patterns of brain activity in young carriers of the APOE-epsilon4 allele. Proc Natl Acad Sci U S A 106:7209-7214.

Gentry LR, Godersky JC, Thompson B (1988) MR imaging of head trauma: review of the distribution and radiopathologic features of traumatic lesions. AJR Am J Roentgenol 150:663-672.

Gilbert SJ, Dumontheil I, Simons JS, Frith CD, Burgess PW (2007) Comment on "Wandering minds: the default network and stimulus-independent thought." Science 317:43, author reply 43.

Good CD, Johnsrude IS, Ashburner J, Henson RN, Friston KJ, Frackowiak RS (2001) A voxel-based morphometric study of ageing in 465 normal adult human brains. Neuroimage 14:21-36.

Greicius M (2008) Resting-state functional connectivity in neuropsychiatric disorders. Curr Opin Neurol 21:424-430.

Greicius MD, Supekar K, Menon V, Dougherty RF (2009) Resting-state functional connectivity reflects structural connectivity in the default mode network. Cereb Cortex 19:72-78.

Grier RA, Warm JS, Dember WN, Matthews G, Galinsky TL, Parasuraman R (2003) The vigilance decrement reflects limitations in effortful attention, not mindlessness. Hum Factors 45:349-359.

Grottick AJ, Higgins GA (2002) Assessing a vigilance decrement in aged rats: effects of pre-feeding, task manipulation, and psychostimulants. Psychopharmacology (Berl) 164:33-41.

Gusnard DA, Akbudak E, Shulman GL, Raichle ME (2001) Medial prefrontal cortex and self-referential mental activity: relation to a default mode of brain function. Proc Natl Acad Sci U S A 98:4259-4264.

Hagmann P, Cammoun L, Gigandet X, Meuli R, Honey CJ, Wedeen VJ, Sporns O (2008) Mapping the structural core of human cerebral cortex. PLoS Biol 6:e159.

Hampson M, Driesen NR, Skudlarski P, Gore JC, Constable RT (2006) Brain connectivity related to working memory performance. J Neurosci 26:13338-13343.

Hayden BY, Smith DV, Platt ML (2009) Electrophysiological correlates of default-mode processing in macaque posterior cingulate cortex. Proc Natl Acad Sci U S A 106:5948-5953.

Hua K, Zhang J, Wakana S, Jiang H, Li X, Reich DS, Calabresi PA, Pekar JJ, van Zijl PC, Mori S (2008) Tract probability maps in stereotaxic spaces: analyses of white matter anatomy and tract-specific quantification. Neuroimage 39:336-347.

Jenkinson M, Smith S (2001) A global optimisation method for robust affine registration of brain images. Med Image Anal 5:143-156.

Kelly AM, Uddin LQ, Biswal BB, Castellanos FX, Milham MP (2008) Competition between functional brain networks mediates behavioral variability. Neuroimage 39:527-537.

Kim YH, Gitelman DR, Nobre AC, Parrish TB, LaBar KS, Mesulam MM (1999) The large-scale neural network for spatial attention displays multifunctional overlap but differential asymmetry. Neuroimage 9:269-277.

Kim YH, Yoo WK, Ko MH, Park CH, Kim ST, Na DL (2009) Plasticity of the attentional network after brain injury and cognitive rehabilitation. Neurorehabil Neural Repair 23:468-477.

Kinnunen KM, Greenwood R, Powell JH, Leech R, Hawkins PC, Bonnelle V, Patel MC, Counsell SJ, Sharp DJ (2010) White matter damage and cognitive impairment after traumatic brain injury. Brain 134:449-463.

Langner R, Willmes K, Chatterjee A, Eickhoff SB, Sturm W (2010) Energetic effects of stimulus intensity on prolonged simple reaction-time performance. Psychol Res 74:499-512. 
Leech R, Kamourieh S, Beckmann CF, Sharp DJ (2011) Fractionating the default mode network: distinct contributions of the ventral and dorsal posterior cingulate cortex to cognitive control. J Neurosci 31:3217-3224.

Levine B, Fujiwara E, O’Connor C, Richard N, Kovacevic N, Mandic M, Restagno A, Easdon C, Robertson IH, Graham SJ, Cheung G, Gao F, Schwartz ML, Black SE (2006) In vivo characterization of traumatic brain injury neuropathology with structural and functional neuroimaging. J Neurotrauma 23:1396-1411.

Lisper HO, Tornros J (1974) Effects of inter-signal interval regularity on increase in reaction time in a one hour auditory monitoring task. Acta Psychol (Amst) 38:455-460.

Lowenstein DH (2009) Traumatic brain injury: a glimpse of order among the chaos? Ann Neurol 66:A7-A8.

Malec JF, Brown AW, Leibson CL, Flaada JT, Mandrekar JN, Diehl NN, Perkins PK (2007) The mayo classification system for traumatic brain injury severity. J Neurotrauma 24:1417-1424.

Malhotra P, Coulthard EJ, Husain M (2009) Role of right posterior parietal cortex in maintaining attention to spatial locations over time. Brain 132:645-660.

Manly T, Robertson IH, Galloway M, Hawkins K (1999) The absent mind: further investigations of sustained attention to response. Neuropsychologia 37:661-670.

Mason MF, Norton MI, Van Horn JD, Wegner DM, Grafton ST, Macrae CN (2007) Wandering minds: the default network and stimulus-independent thought. Science 315:393-395.

Millis SR, Rosenthal M, Novack TA, Sherer M, Nick TG, Kreutzer JS, High WM Jr, Ricker JH (2001) Long-term neuropsychological outcome after traumatic brain injury. J Head Trauma Rehabil 16:343-355.

Mori S, Wakana S, van Zijl P, Nagae-Poetscher LM (2005) MRI atlas of human white matter. Amsterdam: Elsevier.

Oakes TR, Fox AS, Johnstone T, Chung MK, Kalin N, Davidson RJ (2007) Integrating VBM into the General Linear Model with voxelwise anatomical covariates. Neuroimage 34:500-508.

Pattyn N, Neyt X, Henderickx D, Soetens E (2008) Psychophysiological investigation of vigilance decrement: boredom or cognitive fatigue? Physiol Behav 93:369-378.

Paus T, Zatorre RJ, Hofle N, Caramanos Z, Gotman J, Petrides M, Evans AC (1997) Time-related changes in neural systems underlying attention and arousal during the performance of an auditory vigilance task. J Cogn Neurosci 9:392-408.

Povlishock JT, Katz DI (2005) Update of neuropathology and neurological recovery after traumatic brain injury. J Head Trauma Rehabil 20:76-94.

Price CJ, Crinion J, Friston KJ (2006) Design and analysis of fMRI studies with neurologically impaired patients. J Magn Reson Imaging 23:816-826.

Pyka M, Beckmann CF, Schoning S, Hauke S, Heider D, Kugel H, Arolt V, Konrad C (2009) Impact of working memory load on FMRI resting state pattern in subsequent resting phases. PLoS One 4:e7198.

Raichle ME (2010) Two views of brain function. Trends Cogn Sci 14:180-190.

Raichle ME, MacLeod AM, Snyder AZ, Powers WJ, Gusnard DA, Shulman GL (2001) A default mode of brain function. Proc Natl Acad Sci U S A 98:676-682.

Rasmussen IA, Xu J, Antonsen IK, Brunner J, Skandsen T, Axelson DE, Berntsen EM, Lydersen S, Haberg A (2008) Simple dual tasking recruits prefrontal cortices in chronic severe traumatic brain injury patients, but not in controls. J Neurotrauma 25:1057-1070.

Reitan R (1958) The validity of the Trail Making test as an indicator of organic brain damage. Percept Mot Skills 8:271-276.

Robertson IH, Manly T, Andrade J, Baddeley BT, Yiend J (1997) 'Oops!': performance correlates of everyday attentional failures in traumatic brain injured and normal subjects. Neuropsychologia 35:747-758.
Rueckert L, Grafman J (1996) Sustained attention deficits in patients with right frontal lesions. Neuropsychologia 34:953-963.

Sarter M, Givens B, Bruno JP (2001) The cognitive neuroscience of sustained attention: where top-down meets bottom-up. Brain Res Brain Res Rev 35:146-160.

Scheibel RS, Newsome MR, Steinberg JL, Pearson DA, Rauch RA, Mao H, Troyanskaya M, Sharma RG, Levin HS (2007) Altered brain activation during cognitive control in patients with moderate to severe traumatic brain injury. Neurorehabil Neural Repair 21:36-45.

Scheid R, Preul C, Gruber O, Wiggins C, von Cramon DY (2003) Diffuse axonal injury associated with chronic traumatic brain injury: evidence from $\mathrm{T} 22^{*}$-weighted gradient-echo imaging at $3 \mathrm{~T}$. AJNR Am J Neuroradiol 24:1049-1056.

Schmahmann JD, Pandya DN (2006) Fiber pathways of the brain. Oxford, UK: Oxford UP.

Seeley WW, Menon V, Schatzberg AF, Keller J, Glover GH, Kenna H, Reiss AL, Greicius MD (2007) Dissociable intrinsic connectivity networks for salience processing and executive control. J Neurosci 27:2349-2356.

Singh KD, Fawcett IP (2008) Transient and linearly graded deactivation of the human default-mode network by a visual detection task. Neuroimage 41:100-112.

Smith SM (2002) Fast robust automated brain extraction. Hum Brain Mapp 17:143-155.

Smith SM, Jenkinson M, Woolrich MW, Beckmann CF, Behrens TE, Johansen-Berg H, Bannister PR, De Luca M, Drobnjak I, Flitney DE, Niazy RK, Saunders J, Vickers J, Zhang Y, De Stefano N, Brady JM, Matthews PM (2004) Advances in functional and structural MR image analysis and implementation as FSL. Neuroimage 23 [Suppl 1]:S208-S219.

Smits M, Dippel DW, Houston GC, Wielopolski PA, Koudstaal PJ, Hunink MG, van der Lugt A (2009) Postconcussion syndrome after minor head injury: brain activation of working memory and attention. Hum Brain Mapp 30:2789-2803.

Stuss DT, Stethem LL, Hugenholtz H, Picton T, Pivik J, Richard MT (1989) Reaction time after head injury: fatigue, divided and focused attention, and consistency of performance. J Neurol Neurosurg Psychiatry 52:742-748.

Takahashi M, Iwamoto K, Fukatsu H, Naganawa S, Iidaka T, Ozaki N (2010) White matter microstructure of the cingulum and cerebellar peduncle is related to sustained attention and working memory: a diffusion tensor imaging study. Neurosci Lett 477:72-76.

van den Heuvel M, Mandl R, Luigjes J, Hulshoff Pol H (2008) Microstructural organization of the cingulum tract and the level of default mode functional connectivity. J Neurosci 28:10844-10851.

Wechsler D (1997) Wechsler memory scale — third edition: administration and scoring manual. San Antonio, TX: Psychological Corporation.

Wechsler D (1999) WASI: Wechsler abbreviated scale of intelligence. San Antonio, TX: Psychological Corporation.

Weissman DH, Roberts KC, Visscher KM, Woldorff MG (2006) The neural bases of momentary lapses in attention. Nat Neurosci 9:971-978.

Whyte J, Polansky M, Fleming M, Coslett HB, Cavallucci C (1995) Sustained arousal and attention after traumatic brain injury. Neuropsychologia 33:797-813.

Zhang S, Li CS (2010) A neural measure of behavioral engagement: taskresidual low-frequency blood oxygenation level-dependent activity in the precuneus. Neuroimage 49:1911-1918.

Zhang Y, Brady M, Smith S (2001) Segmentation of brain MR images through a hidden Markov random field model and the expectationmaximization algorithm. IEEE Trans Med Imaging 20:45-57.

Zuo XN, Kelly C, Adelstein JS, Klein DF, Castellanos FX, Milham MP (2010) Reliable intrinsic connectivity networks: test-retest evaluation using ICA and dual regression approach. Neuroimage 49:2163-2177. 